\title{
The Anticonvulsant Response to Valproate in Kindled Rats Is Correlated with Its Effect on Neuronal Firing in the Substantia Nigra Pars Reticulata: A New Mechanism of Pharmacoresistance
}

\author{
Kathrin Töllner, ${ }^{1,2}$ Saskia Wolf, ${ }^{1}$ Wolfgang Löscher, ${ }^{1,2}$ and Manuela Gernert ${ }^{1,2}$ \\ ${ }^{1}$ Department of Pharmacology, Toxicology, and Pharmacy, University of Veterinary Medicine Hannover, and ${ }^{2}$ Center for Systems Neuroscience, 30559 \\ Hannover, Germany
}

Resistance to antiepileptic drugs (AEDs) is a major problem in epilepsy treatment. However, mechanisms of resistance are only incompletely understood. We have recently shown that repeated administration of the AED phenytoin allows selecting resistant and responsive rats from the amygdala kindling model of epilepsy, providing a tool to study mechanisms of AED resistance. We now tested whether individual amygdala-kindled rats also differ in their anticonvulsant response to the major AED valproate (VPA) and which mechanism may underlie the different response to VPA. VPA has been proposed to act, at least in part, by reducing spontaneous activity in the substantia nigra pars reticulata $(\mathrm{SNr})$, a main basal ganglia output structure involved in seizure propagation, seizure control, and epilepsy-induced neuroplasticity. Thus, we evaluated whether poor anticonvulsant response to VPA is correlated with low efficacy of VPA on SNr firing rate and pattern in kindled rats. We found (1) that good and poor VPA responders can be selected in kindled rats by repeatedly determining the effect of VPA on the electrographic seizure threshold, and (2) a significant correlation between the anticonvulsant response to VPA in kindled rats and its effect on SNr firing rate and pattern. The less VPA was able to raise seizure threshold, the lower was the VPA-induced reduction of SNr firing rate and the VPA-induced regularity of SNr firing. The data demonstrate for the first time an involvement of the $\mathrm{SNr}$ in pharmacoresistant experimental epilepsy and emphasize the relevance of the basal ganglia as target structures for new treatment options.

\section{Introduction}

Temporal lobe epilepsy (TLE) is a common and often devastating type of epilepsy, which is often resistant to antiepileptic drugs (AEDs) (French, 2007). Pharmacoresistance to AEDs is known to be a multifactorial process including genetic, disease-related, and drug-related mechanisms. Presently, four main hypotheses are discussed for pharmacoresistance in epilepsy: (1) the gene variant hypothesis, suggesting genetic polymorphisms affecting the response to AEDs; (2) the multidrug transporter hypothesis, suggesting that increased expression of drug efflux transporters decreases brain AED levels; (3) the target hypothesis, suggesting AED target alterations in epileptogenic brain tissue; and (4) the network hypothesis, suggesting that "epileptic networks" differ

\footnotetext{
Received May 19, 2011; revised Sept. 15, 2011; accepted Sept. 22, 2011.

Author contributions: M.G. designed research; K.T. and S.W. performed research; K.T. and M.G. analyzed data; K.T., W.L., and M.G. wrote the paper.

This work was supported by Deutsche Forschungsgemeinschaft Grant GE 1103/6. We are deeply grateful to Dr. Yoshiki Kaneoke (Department of Integrative Physiology, National Institute for Physiological Sciences, Okazaki, Japan) for providing his burst analysis software and for help with the analysis. The assistance of Nicole Ernst, Martina Gramer, Maria Hausknecht, Doris Möller, Christian Rathert, and Michael Weißing is gratefully acknowledged.

Correspondence should be addressed to Dr. Manuela Gernert, Department of Pharmacology, Toxicology, and Pharmacy, University of Veterinary Medicine Hannover, Bünteweg 17, 30559 Hannover, Germany. E-mail: manuela.gernert@tiho-hannover.de.

DOI:10.1523/JNEUROSCI.2506-11.2011

Copyright $\odot 2011$ the authors $\quad 0270-6474 / 11 / 3116423-12 \$ 15.00 / 0$
}

between pharmacoresistant and pharmacosensitive patients (Löscher and Potschka, 2005; Schmidt and Löscher, 2005; Remy and Beck, 2006; Szoeke et al., 2006).

Epileptic networks in experimental epilepsy are characterized by widespread network changes in and close to the seizure focus but also in remote brain structures including the basal ganglia. The substantia nigra pars reticulata $(\mathrm{SNr})$, a main basal ganglia output structure, is thought to be crucially involved in the propagation and modulation of different types of experimental seizures including complex-partial seizures as observed in TLE (Iadarola and Gale, 1982; Garcia-Cairasco and Sabbatini, 1983; Le Gal La Salle et al., 1983; Albala et al., 1984; De Sarro et al., 1984, 1986, 1991; McNamara et al., 1984; Moshé and Albala, 1984; Sperber et al., 1987; Moshé et al., 1992; Gale et al., 2008). Using in vivo recordings, numerous plastic network changes were shown for basal ganglia structures including the $\mathrm{SNr}$ in amygdalakindled rats as a model for TLE (Gernert et al., 2004; Nolte et al., 2006; Kücker et al., 2010). Several AEDs including valproate (VPA) are known to reduce firing rates of $\mathrm{SNr}$ neurons in naive rats (Kerwin et al., 1980; Waszczak et al., 1986; Farrant and Webster, 1989; Löscher et al., 1995; Rohlfs et al., 1996). It was discussed that this may be an important mechanism through which some AEDs exert their anticonvulsant properties. More recent data of our group indicated that epilepsy-induced alterations of 


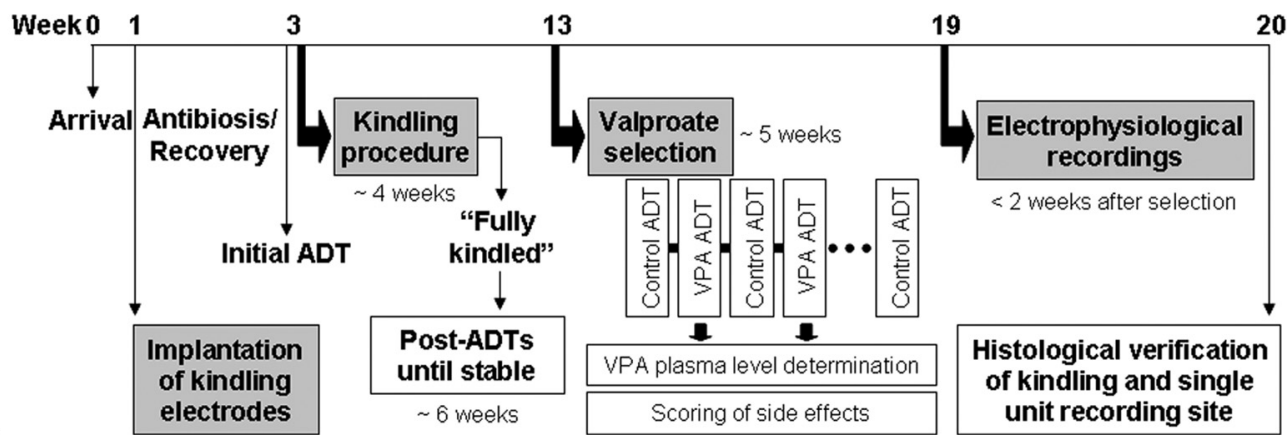

Figure 1. Study design. Adult female Wistar rats were implanted with electrodes for kindling stimulation and recording of afterdischarges. Initially, the stimulation threshold for eliciting afterdischarges (initial ADT, afterdischarge threshold) was determined for each animal followed by daily constant current stimulations. In fully kindled rats, the stimulation threshold for eliciting afterdischarges (post-ADT) was determined for each animal until reproducible postkindling (control) ADTs were observed. For the selection procedure, VPA was administered (200 mg/kg, i.p.) 30 min before ADT determinations. Scorings of side effects induced by VPA included determination of severity of ataxia, hypolocomotion/sedation, abdominal muscle tone, amount of wet dog shake behavior, body temperature, and rotarod performance. VPA trials were repeated at least four times alternating with control trials with injection of saline before stimulation. Within 2 weeks after the last determination of ADT and $24 \mathrm{~h}$ after a further kindled seizure was elicited, extracellular single-unit recordings of nondopaminergic, presumably GABAergic nigral neurons were performed in each rat. Finally, rats were deeply anesthetized with chloral hydrate and transcardially perfused to process the brains for histological verification of the kindling site and the recording site.

basal ganglia networks are able to change the nigral sensitivity to AEDs. Gernert et al. (2004) showed that VPA is significantly less effective in reducing $\mathrm{SNr}$ firing rates in kindled compared with naive control rats. However, this previous study did not determine whether the effect of VPA on SNr firing is correlated with its anticonvulsant response. We have previously shown that kindled rats differ in their individual anticonvulsant response to the AED phenytoin (Löscher, 2006). This allows to select responsive and resistant rats (i.e., responders and nonresponders), thus permitting use of these subgroups in the search for mechanisms of pharmacoresistance in TLE.

We now tested whether individual kindled rats also differ in their anticonvulsant response to VPA and whether the different anticonvulsant efficacy is correlated with interindividual differences in the sensitivity of SNr neurons to VPA. Such correlation would enhance our understanding of the mechanism of action of VPA and also, for the first time, implicate a role of the $\mathrm{SNr}$ in mechanisms of AED resistance.

\section{Materials and Methods}

An overview of the study design is illustrated in Figure 1. This design was performed in four consecutive batches of 10-15 animals each batch. Because comparison of afterdischarge threshold (ADT) increases after VPA did not reveal statistical differences between the batches (ANOVA, $p=0.631$ ), the results of all four batches were pooled for further analysis.

Animals. Adult female Wistar rats (Harlan Winkelmann) were purchased at a body weight of 200-220 g. The total number of animals purchased from the breeder in four consecutive batches and used for the present experiments was 50 . The rats were housed individually and kept under controlled environmental conditions with a $12 \mathrm{~h}$ light/dark cycle, lights on at 6:00 A.M., for 2 weeks before experiments started. Standard laboratory chow (Altromin 1324 standard diet) and tap water were allowed ad libitum. Cages were changed once weekly and standard bedding (Altromin soft wood granulate) was used. Female rats were housed without males to keep them acyclic or asynchronous with respect to their estrous cycle (Kücker et al., 2010). All experiments were performed in accordance with the European Communities Council Directive of November 24, 1986 (86/609/EEC), and were formally approved by the animal subjects review board of our institution. All efforts were made to minimize the number of animals and their suffering.

Kindling. For surgical implantation of kindling electrodes, 50 rats were anesthetized with chloral hydrate $(360 \mathrm{mg} / \mathrm{kg}$, i.p. $)$ and treated with the analgesic drug buprenorphine $(0.045 \mathrm{mg} / \mathrm{kg}$, i.m. $)$. A Teflon-isolated bipolar stainless-steel electrode was aimed at the right basolateral amygdala (BLA). The electrode was stereotaxically implanted for kin- dling and for recording of afterdischarges (Fig. 1). The stereotaxic coordinates in millimeters relative to bregma according to the atlas of Paxinos and Watson (2007) were posterior, 2.2; lateral, 4.8; and ventral, $8.5 \mathrm{~mm}$. The incisor bar was set at $-3.3 \mathrm{~mm}$. A stainless-steel screw placed above the left parietal cortex served as grounding. Bipolar and grounding electrodes were connected to plugs. Additional skull screws and dental acrylic cement anchored the entire headset. The skull above the right $\mathrm{SNr}$ was kept free from acrylic cement.

Electrical stimulation via the kindling electrode was initiated after a recovery period of 2 weeks after surgery. From the 50 rats implanted with amygdala electrodes, kindling could only be started with 42 rats because of problems such as loss of headset or failure of grounding electrode. Kindling was performed at the same time of the day (between 9:00 and 12:00 A.M.) to avoid intraday variance between animals. Initially, the stimulation threshold for eliciting afterdischarges (initial ADT, afterdischarge threshold) was determined for each animal using an ascending stair step procedure as described previously (Gernert and Löscher, 2001). From the next day on, constant current stimulations (500 $\mu \mathrm{A}, 1 \mathrm{~ms}$, monophasic square-wave pulses, 50 pulses/s for $1 \mathrm{~s}$ ) were delivered once daily to the kindling site until at least 10 fully kindled seizures [secondarily generalized stage 5 seizures classified according to Racine (1972)] were elicited. In fully kindled rats, the ADT was determined for each animal 3-5 d after kindling as described for the initial ADT and repeated at intervals of at least $2 \mathrm{~d}$ until all rats exhibited a reproducible postkindling (control) ADT.

The following parameters were recorded during kindling and during determination of ADTs: seizure severity, seizure duration, and afterdischarge duration. Seizure severity was classified behaviorally according to Racine (1972): stage 1, immobility, slight facial clonus (eye closure, twitching of vibrissae, sniffing); stage 2 , head nodding associated with more severe facial clonus; stage 3, clonus of one forelimb; stage 4, rearing, often accompanied by bilateral forelimb clonus; stage 5 , tonic-clonic seizure accompanied by loss of balance and falling. Seizure duration was the time from beginning of electrical stimulation until the end of motor seizures. Afterdischarge duration was the total duration of EEG spikes with amplitudes of at least twice the amplitude of the prestimulus recording and a frequency of at least $1 / \mathrm{s}$.

Parameters for evaluation of the kindling development were number of days until first stage 5 seizure, number of days until fully kindled state, cumulative seizure duration, and cumulative afterdischarge duration for stages 2, 4, and 5. Stage 3 was usually skipped and therefore not evaluated.

Effects of VPA on kindled seizures. After reproducible ADTs were obtained (Fig. 1), VPA was administered at a dose $(200 \mathrm{mg} / \mathrm{kg}$, i.p.) that had previously been shown to increase the ADT in kindled rats (Löscher et al., 1993), to enhance GABA turnover in the substantia nigra (Löscher, 1989), and to reduce single-unit firing in the $\mathrm{SNr}$ (Rohlfs et al., 1996). To 
avoid intolerable severe side effects such as pronounced sedation, we did not increase this dose in the course of the experiments. Pretreatment time (30 min) was chosen based on preliminary experiments and on previous studies in kindled rats (Löscher et al., 1993). In the preliminary experiments, we compared VPA plasma levels (see below) as well as the anticonvulsant efficacy (elevation of ADT) at time points of 15 and $30 \mathrm{~min}$, respectively, after intraperitoneal injection of $200 \mathrm{mg} / \mathrm{kg}$ VPA. There were no differences between these two pretreatment times (not illustrated). Determination of ADT was started at a current that was three $20 \%$ steps below the individual predrug (control) ADT. The current was elevated at $1 \mathrm{~min}$ intervals of $\sim 20 \%$ of the previous current, so that the current intensity of the control threshold was delivered $30 \mathrm{~min}$ after injection of VPA. Current elevation was continued until an afterdischarge of at least $3 \mathrm{~s}$ duration was recorded. Usually, fully kindled rats showed generalized motor seizures at the ADT. Otherwise, the current was further elevated up to the generalized seizure threshold (GST) or up to a maximum current intensity of $840 \mu \mathrm{A}$. These trials with VPA were performed at least four times in each rat to determine the reproducibility and intertrial variability of the anticonvulsant effect of VPA in each rat. Intervals between two drug injections were at least $5 \mathrm{~d}$ to avoid drug accumulation or development of tolerance. Control ADTs were determined $30 \mathrm{~min}$ after intraperitoneal injection of saline $2-5 \mathrm{~d}$ before and after each VPA injection.

Following determination of ADT after injection of VPA, blood samples were immediately withdrawn by retrobulbar venous plexus puncture after local anesthesia with tetracaine hydrochloride (2\%) for drug analysis in plasma. Blood samples were anticoagulated with EDTA (5 $\mathrm{mmol} / \mathrm{ml}$ whole blood), centrifuged for $2.5 \mathrm{~min}$ at $12,000 \mathrm{rpm}$, and stored at $-20^{\circ} \mathrm{C}$ until analysis. VPA concentrations were determined by HPLC with ultraviolet detection (Potschka and Löscher, 2001). Following administration of VPA, drug plasma levels were typically $>250 \mu \mathrm{g} / \mathrm{ml}$ (see Results) (i.e., plasma levels known to be associated with anticonvulsant activity of VPA in rodent models) (Löscher, 2007). Experiments in which plasma levels were $<250 \mu \mathrm{g} / \mathrm{ml}$ ( $6 \%$ of injections), indicating insufficient drug absorption and/or erroneous drug injection, were repeated. In this respect, it is important to note that plasma concentrations associated with significant anticonvulsant activity of single doses of VPA in rodent models $(>250 \mu \mathrm{g} / \mathrm{ml})$ are much higher than the therapeutic plasma concentration range of VPA $(50-100 \mu \mathrm{g} / \mathrm{ml})$ in epilepsy patients undergoing chronic administration of VPA (Löscher, 2007).

Rats that did not exhibit reproducible control ADTs or lost their electrode assembly before at least four trials with VPA were completed, or that showed misplaced electrode location during later histological analysis were excluded from final analysis, so that the group size for evaluation of the anticonvulsant efficacy of VPA in kindled rats was reduced to 27 .

For calculation of the effect of VPA on ADT, the individual mean of at least four control ADT values (and other parameters at seizure threshold determinations after intraperitoneal injection of saline) was set at $100 \%$ and compared with the individual mean of at least four drug values (and other parameters at seizure threshold determinations after intraperitoneal injection of VPA).

Quantification of side effects of VPA in kindled rats. A thorough investigation of side effects induced by VPA was performed starting 10 and 23 min after VPA injection (i.e., before determination of ADT) in 20 rats. The following behavioral features were investigated consecutively. Severity of ataxia, hypolocomotion/sedation, and abdominal muscle relaxation were determined using a score as follows: 0 , not present; 1 , equivocal; 2 , present; 3 , intensive. Animals were placed in an open field and observed for $\sim 1 \mathrm{~min}$ to score ataxia and hypolocomotion/sedation. Reduction of abdominal muscle tone was evaluated by palpation at the end of the observation period. Body temperature was measured before VPA injection, $10 \mathrm{~min}$, and $23 \mathrm{~min}$ after VPA injection using a rectal probe. To further test motor coordination and balance, animals had to perform the rotarod task 10 and $23 \mathrm{~min}$ after VPA injection. To pass this test, animals had to balance on a rotating rod $(8 \mathrm{rpm})$ without falling off for at least $1 \mathrm{~min}$ in one of three trials. Wet dog shake behavior was counted in blocks of 5 min starting with the injection of VPA up to the electrical stimulation.
Electrophysiology. Within 2 weeks after the last determination of ADT, extracellular single-unit recording of $\mathrm{SNr}$ neurons ipsilateral to the kindling electrode was performed. One day before electrophysiological measurements, rats were stimulated once again by using the same parameters as were used during the kindling procedure to ensure that electrophysiological readings were performed at the same time interval after a seizure in all rats. Extracellular single-unit recordings were performed using standard in vivo techniques as described in detail previously (Gernert et al., 2004; Nolte et al., 2006; Kücker et al., 2010). Briefly, the animals initially received methohexital ( $55 \mathrm{mg} / \mathrm{kg}$, i.p.) and fentanyl $(0.05 \mathrm{mg} / \mathrm{kg}$, i.p.) to achieve surgical anesthesia for cannulation of the vena femoralis, followed by intravenous infusion of the short-acting anesthetic propofol $\left(10-20 \mathrm{mg} \cdot \mathrm{kg}^{-1} \cdot \mathrm{h}^{-1}\right)$ during surgical preparation. During recordings, the animals received fentanyl $\left(0.05 \mathrm{mg} \cdot \mathrm{kg}^{-1} \cdot \mathrm{h}^{-1}\right.$, i.v. $)$ and gallamine $\left(15 \mathrm{mg} \cdot \mathrm{kg}^{-1} \cdot \mathrm{h}^{-1}\right.$, i.v.). This medication has previously been shown to not alter the spontaneous discharge rate of GABAergic nigral neurons when compared with conscious animals (Löscher et al., 1995). Short hindpaw pinches were applied to test for signs of stress or pain. In occasional cases, in which signs of stress or pain in response to the hindpaw pinch occurred, the dosage of intravenously administered medication was corrected to higher values. The rats were artificially ventilated with room air in a volume-constant mode (rate, 65/min; tidal volume, 2.5-3.5 ml; expired $\mathrm{CO}_{2}$ level, $\sim 2 \%$ ). Level of vigilance was assessed indirectly by monitoring of heart rate, blood pressure, and expired $\mathrm{CO}_{2}$ level, which all had to be stable (no fluctuations and no decline) to include an animal into final evaluation of data. To verify stability of vital parameters, heart rate and arterial blood pressure (measured via the arteria femoralis) were continuously monitored and the body temperature was maintained at $37^{\circ} \mathrm{C}$ by heating pad. No animal did show episodic tachycardia as a sign of higher level of vigilance during electrophysiological recording. Single-unit recordings were begun no earlier than $1 \mathrm{~h}$ after the last injection of propofol, assuming that methohexital and propofol had been eliminated from the brain at this time.

Single-barrel extracellular recording microelectrodes were pulled (Narishige PE-2 vertical puller) from a filamented glass electrode (Hilgenberg), and the tip was broken back to an external diameter of $\sim 10-20$ $\mu \mathrm{m}$. The electrodes were backfilled with horseradish peroxidase (3\%) in Tris-buffered saline for marking the tip location. This procedure yielded electrodes with impedances of 2-5 M $\Omega$ when tested ex vivo at $1200 \mathrm{~Hz}$. The microelectrode was directed stereotaxically through a drilled skull hole to the SNr ipsilateral to the kindling electrode using the following stereotaxic coordinates according to Paxinos and Watson (2007): posterior, 2.2, and lateral, $4.8 \mathrm{~mm}$ relative to bregma. The incisor bar was set at $-3.3 \mathrm{~mm}$. The microelectrode was lowered to just above the target region (7.5 $\mathrm{mm}$ ventral to bregma). Nondopaminergic, presumably GABAergic neurons were identified on the basis of their location and electrophysiological features. They were located within the $\mathrm{SNr}$ and showed biphasic action potentials with durations of $0.6-1.5 \mathrm{~ms}$ and stable discharge rates of typically at least $10 \mathrm{~Hz}$ (Guyenet and Aghajanian, 1978; Waszczak et al., 1986). Dopaminergic neurons are easily recognized and differentiated from nondopaminergic neurons by their longer time course (1.5-4.5 ms) and their lower discharge rates (3-6 Hz) (Deniau et al., 1978; Grace and Bunney, 1979; Löscher et al., 1995).

Initially, the basal activity of a tonically active nondopaminergic, presumably GABAergic neuron was recorded for $5 \mathrm{~min}$. Under continuation of electrophysiological recording, VPA was given slowly (typically over $30 \mathrm{~s}$ ) intravenously at $100 \mathrm{mg} / \mathrm{kg}$ (injection volume, $2 \mathrm{ml} / \mathrm{kg}$ ). This intravenous dose is known to significantly reduce the $\mathrm{SNr}$ discharge rate in naive (Rohlfs et al., 1996) and kindled rats (Gernert et al., 2004) without sustained influence on heart rate and blood pressure, whereas higher intravenous doses of VPA (e.g., $200 \mathrm{mg} / \mathrm{kg}$ ) induce an intolerable longlasting drop in blood pressure (M. Gernert, unpublished data; Löscher et al., 1995; Rohlfs et al., 1996). Intravenous rather than intraperitoneal injection was used to prevent malinjection of VPA in the laborious electrophysiological experiments, which could be performed only once in each rat. Two preconditions had to be fulfilled to choose a neuron for recording and to decide to inject VPA, as well as to later include the sampled neuron into further evaluation: (1) stable vital parameters of the 
animal and (2) a stable spontaneous activity of the neuron before VPA injection.

Standard techniques for amplifying (10,000-fold), filtering (high pass at $300 \mathrm{~Hz}$, low pass at $4000 \mathrm{~Hz}$, Notch at $50 \mathrm{~Hz}$ ), analog-to-digital conversion (sampling rate, $20 \mathrm{kHz}$ ), and processing of extracellular neuronal activity were used by means of the DataWave System (WissTech). Single units were isolated on-line by amplitude threshold and off-line by spike template matching using the cluster analysis module of DataWave System.

The location of the recorded neuron was marked by iontophoretical ejection of a small amount of horseradish peroxidase as adapted from Simons and Land (1987). For this purpose, a pulsed anodal current (7 s on, $7 \mathrm{~s}$ off) of $2.2 \mu \mathrm{A}$ was applied for $2 \mathrm{~min}$. Finally, rats were deeply anesthetized with chloral hydrate and transcardially perfused. The brains were processed for histological verification of the kindling site and the recording site as described in detail previously (Gernert et al., 2004; Nolte et al., 2006; Kücker et al., 2010) (Fig. 1). Only neurons located in the SNr were used for further evaluation of data.

Additionally, four age-matched controls (kindled rats, treated with VPA before ADT determinations) were injected with saline $(0.9 \%)$ instead of VPA during electrophysiological measurements.

Several rats undergoing the electrophysiological measurements could not be used for final analysis of data because neurons could not be reliably identified, blood pressure was not stable during the experiment, artifacts could not be excluded, the recording electrode was misplaced, or the kindling electrode head set prevented a proper stereotaxic lowering of the microelectrode. Thus, final group size was reduced to seven rats (one neuron per animal).

Evaluation of discharge rate and pattern. Spontaneous discharge rates of neurons (basal activity before VPA injection) were averaged over 5 min recording time for each neuron (mean predrug value). For evaluation of VPA-induced effect on spontaneous firing rate, mean 1 min bins of firing rates after injection of VPA were calculated relative to the mean predrug value, which was set at $100 \%$. For comparison between different animals, the percentage of reduction of firing rates at the individual time of maximum depression within $20 \mathrm{~min}$ after intravenous injection of 100 $\mathrm{mg} / \mathrm{kg}$ VPA was calculated. The time window of $20 \mathrm{~min}$ was chosen based on previous experiments, in which we investigated recording times up to $50 \mathrm{~min}$ after intravenous injection of $100 \mathrm{mg} / \mathrm{kg}$ VPA (Löscher et al., 1995; Rohlfs et al., 1996). In these previous studies, the maximum inhibitory effect of VPA was reached rapidly within a few minutes after injection and was rather long-lasting, although occasionally a recovery of firing rate was observed. This is in line with the rapid penetration of VPA into the brain (but the short half-life in most species), which induces most marked effects typically shortly (i.e., $2-15 \mathrm{~min}$ ) after parenteral (intravenous or intraperitoneal) injection (for review, see Löscher, 2002).

Differences in the regularity of SNr firing before and after VPA injection were determined by comparing interspike interval histogram (ISIH) parameters similar to the method described previously (Gernert et al., 2004; Nolte et al., 2006; Kücker et al., 2010). ISIHs were calculated for a time period of $5 \mathrm{~min}$ before and after injection of VPA. The $5 \mathrm{~min}$ time period after injection of VPA was chosen around the time point of maximum firing rate reduction within $20 \mathrm{~min}$ after VPA injection. The resolution was $1 \mathrm{~ms}$ and intervals up to $2000 \mathrm{~ms}$ were considered. The ISIHs were produced with an event interval histogram module (DataWave). The degree of regularity of neuronal discharge was determined by calculating the coefficient of variation, the kurtosis, and the skewness. The coefficient of variation is defined as the ratio between SD and mean interspike interval. The kurtosis (fourth moment normalized by dividing by squared variance) of a distribution reflects its degree of peakedness relative to the length and size of its tails. A kurtosis of zero reflects a normal distribution, positive values a sharp peak (leptokurtic), and negative values a flat shape (platykurtic). The kurtosis provides information on the regularity of spiking (i.e., the lower the kurtosis, the more irregular a neuron is discharging). Differences to the Gaussian distribution were also determined by evaluation of the skewness. A skewness value of zero reflects a symmetric distribution, positive values reflect a positively skewed shape of ISIH, and negative values reflect a negatively skewed shape.

To further characterize the discharge pattern of SNr neurons before and after VPA injection, burst and oscillation detection algorithms developed and described in detail by Kaneoke and Vitek (1996) were used. Dr. Yoshiki Kaneoke (Department of Integrative Physiology, National Institute for Physiological Sciences, Okazaki, Japan) kindly provided the burst and oscillation detection programs for our data evaluation. The burst detection method is based on detecting changes in discharge rate over small time intervals defined as the reciprocal of the mean discharge rate. Here, 2 min intervals before (minutes 3 and 4 after beginning of recordings) and after injection of VPA (time point of maximum firing rate reduction within $20 \mathrm{~min}$ after VPA injection) were used to calculate the discharge density and to construct a discharge density histogram. The discharge density histograms were plotted with the range from zero to maximum density with a bin width of unity. According to Kaneoke and Vitek (1996), it is assumed that a spike train contains burst periods when its discharge density histogram is significantly positively skewed and different from a Poisson distribution with a mean of 1.0. The oscillation detection method is based on detecting the presence of multiple frequencies by using autocorrelograms and evaluates the significances of frequencies detected. Bin widths of autocorrelograms were set individually for each 2 min spike train with a probability of $>0.5$ spikes in a bin $(100$ $\mathrm{ms}$ at maximum). The lag time for autocorrelograms was set to be 2000 ms. ISIH bin width was $10 \mathrm{~ms}$ ( $1000 \mathrm{~ms}$ at maximum for width and height).

Drugs. Chloral hydrate (Merck) was dissolved in sterile saline. The commercial solution of buprenorphine hydrochloride (Temgesic; Essex Pharma) was mixed 1:1 with sterile saline to increase injection volume. VPA (Orfiril; $100 \mathrm{mg}$ sodium VPA; Desitin) was used as commercial solution for intraperitoneal injections (injection volume, $2 \mathrm{ml} / \mathrm{kg}$ ) or dissolved 1:1 with sterile saline to increase the lower dose of the intravenous injection to an injection volume of $2 \mathrm{ml} / \mathrm{kg}$ as well. Methohexital (Brevimytal-Natrium, 500 mg; Hikma Pharma) was dissolved in sterile saline to obtain a concentration of $11 \mathrm{mg} / \mathrm{ml}$ and stored at $4^{\circ} \mathrm{C}$. Propofol (Disoprivan, 1\%; Zeneca) was used as commercial solution. Gallamine (Gallamine triethiodide; Sigma-Aldrich) was dissolved in sterile saline and was mixed 1:1 with the commercial solution of fentanyl (FentanylJanssen; Janssen). Both drugs were coadministered during recordings. Tetracaine hydrochloride (Caesar \& Loretz) was dissolved in distilled water to obtain a $2 \%$ solution.

Statistics. Elevation of ADTs and reduction of firing rates were normally distributed. Thus, effects of VPA on ADTs and on SNr firing rates were verified with a paired $t$ test. Evaluation of correlation between effect of VPA on ADTs and on SNr firing rates was tested with Pearson's correlation. Parameters of the kindling development, seizure parameters, side effects, VPA plasma levels, ISIH parameters, and bursting and oscillatory properties were not normally distributed. For these parameters, statistical significances were calculated using nonparametric tests (i.e., the Friedman repeated-measures ANOVA on ranks followed by the Wilcoxon signed rank test). Correlations were evaluated using the Spearman rank order correlation. Significance of group differences between good and poor responders were calculated with the Student's $t$ test for normally distributed parameters (i.e., control ADTs and VPA ADTs) or with the Mann-Whitney $U$ test for not normally distributed parameters (i.e., kindling development, side effects, and VPA plasma levels). All tests were used two-tailed and an error probability of $<5 \%$ was considered significant. Statistical analysis was performed using GraphPad Prism (5.03).

\section{Results \\ Kindling electrode placement and electrophysiological recording sites}

To verify correct placement of electrodes and to assess putative correlations between electrode placement and efficacy of VPA, the placement of kindling electrodes and electrophysiological recording sites were examined histologically after termination of the electrophysiological experiments (Fig. 2). Among the animals that were tested for an effect of VPA on ADT, 22 animals had 

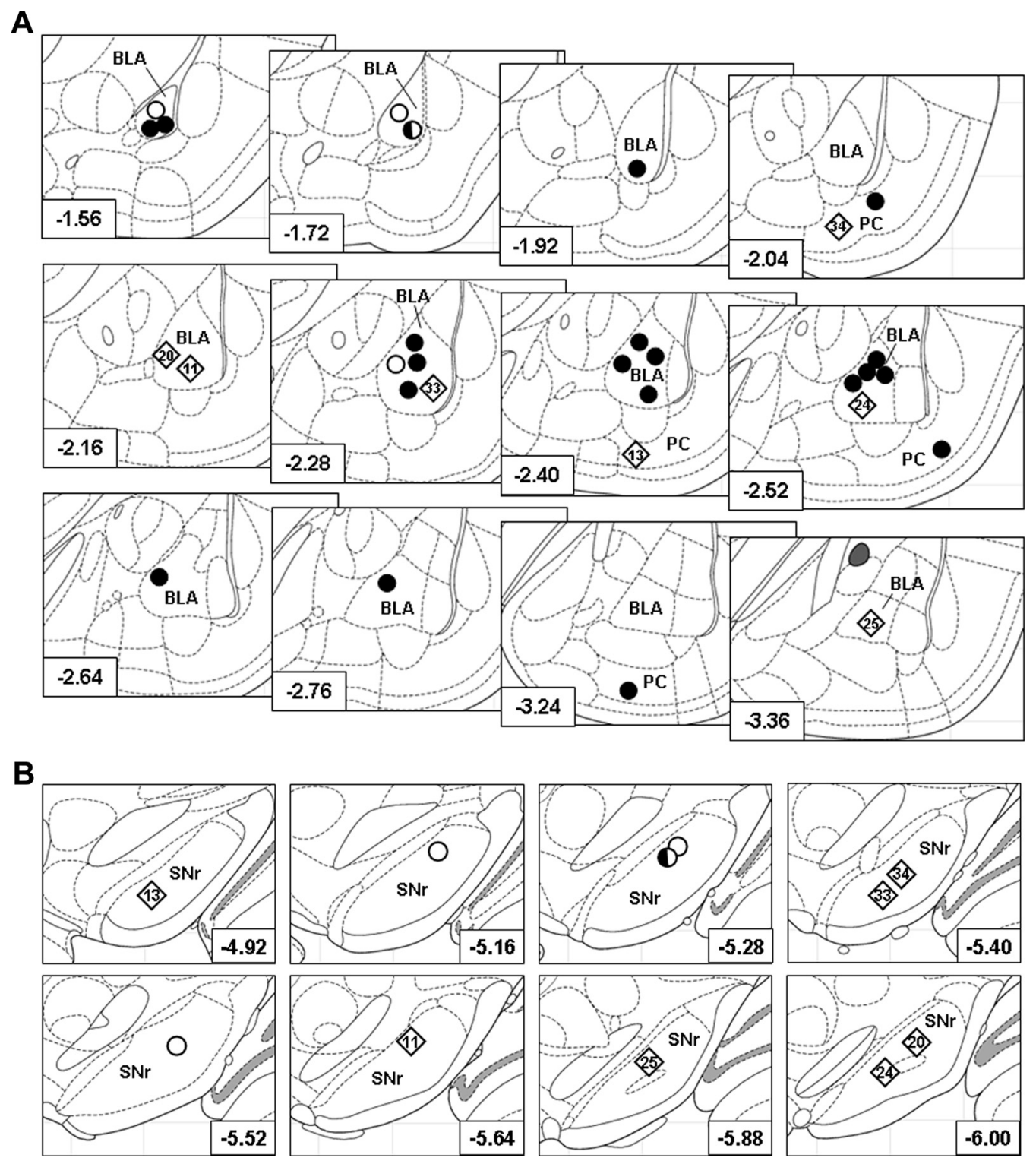

Figure 2. Histologically verified kindling sites and single unit recording sites. $A$, Location of kindling electrodes in the right BLA or the piriform cortex (PC) in 30 rats, drawn on cutouts of coronal sections of the rat brain according to Paxinos and Watson (2007). Filled circles, Animals used for determination of the effect of VPA on kindled ADTs; rhombs, animals used for determination of the effect of VPA on kindled ADTs and for recording of the effect of VPA on SNr neurons (animal names are given within the rhombs to indicate animals that showed a poor or good response to VPA during electrophysiological recordings; compare with Fig. 4); half-filled circle, animal used for determination of the effect of VPA on kindled ADTs and for control recordings with injection of saline during electrophysiological recording; open circles, animals used for control recordings with injection of saline during electrophysiological recording. The distance to bregma in millimeters is given in the left corner of each section. B, Single-unit recording sites in the SNr in animals tested for the efficacy of VPA on SNr firing rates and patterns in kindled rats (rhombs; $n=7$; animal names are given within the rhombs to indicate animals that showed a poor or good response to VPA during electrophysiological recordings; compare with Fig. 4) and saline in control rats (half-filled circle and open circles; together $n=4$ ). The distance to bregma in millimeters is given in the right corner of each cutout of coronal sections of the rat brain according to Paxinos and Watson (2007).

electrode tips in the basolateral amygdaloid nucleus (1.56 to 3.36 $\mathrm{mm}$ posterior to bregma) and five animals had electrode tips in the lamina III of the piriform cortex [2.04-3.24 mm posterior to bregma according to Paxinos and Watson (2007); Fig. 2A]. Because kindling parameters did not differ between rats kindled via BLA and via piriform cortex, we pooled the data from these two subgroups for evaluation of the anticonvulsant efficacy of VPA in kindled rats $(n=27)$. The anticonvulsant efficacy of VPA on ADTs was not correlated with the anterior-posterior location (in millimeters relative to bregma) of kindling electrodes (Pearson's correlation; $r=0.2300 ; p=0.2485$ ), which is similar to previous experiments in phenytoin responders and nonresponders (Ebert et al., 1999).
The effect of VPA on spontaneous $\mathrm{SNr}$ discharge rates was determined in five amygdala-kindled and two piriform cortexkindled rats. The electrophysiologically recorded $\mathrm{SNr}$ neurons were assigned to the anterior $(4.36-5.40 \mathrm{~mm}$ posterior to bregma; $n=3)$ or posterior subregion of the $\mathrm{SNr}(5.52-6.72 \mathrm{~mm}$ posterior to bregma; $n=4)$, respectively [adapted from the study by Gernert et al. (2004)]. Independent of this assignment, a putative correlation between the efficacy of VPA to reduce $\mathrm{SNr}$ firing rate and the anterior-posterior location (in millimeters relative to bregma) of the electrophysiological recording site was tested (Fig. $2 B$ ). Although sample size was small, the data indicate that the efficacy of VPA in reducing $\mathrm{SNr}$ firing rates was neither correlated with the anterior-posterior location of kindling elec- 
Table 1. Effect of valproate on afterdischarge thresholds and seizure parameters

\begin{tabular}{llcccc}
\hline Parameter & Group & Minimum & Median & Maximum & Mean \pm SEM \\
\hline ADT $(\mu \mathrm{A})$ & Control & 29.2 & 64.0 & 145.0 & $72.0 \pm 6.1$ \\
& VPA & 86.3 & 213.3 & 403.3 & $216.0 \pm 17.8^{* * *}$ \\
SS (stage) & Control & 4.5 & 5.0 & 5.0 & $5.0 \pm 0.0$ \\
& VPA & 0 & 1 & 3.3 & $1.20 \pm 0.2^{* * *}$ \\
SD $(s)$ & Control & 35.5 & 56.7 & 75.8 & $55.1 \pm 1.8$ \\
& VPA & 0 & 6.8 & 31.0 & $9.8 \pm 1.6^{* * *}$ \\
ADD $(s)$ & Control & 40.3 & 62.3 & 113.0 & $65.3 \pm 3.5$ \\
& VPA & 3.8 & 11.3 & 28.8 & $12.1 \pm 1.4^{* * *}$ \\
\hline
\end{tabular}

For evaluation of ADTs and seizure parameters at ADTs, the individual means of predrug (control) values were averaged and then compared to the individual means after injection of $200 \mathrm{mg} / \mathrm{kg}$ VPA (at least four control and four VPA trials per rat; $n=27$ rats). All seizure parameters of drug trials differed significantly from seizure parameters of control trials, reflecting the known anticonvulsant efficacy of VPA in kindled rats. SS, Seizure severity; SD, seizure duration; ADD, afterdischarge duration.

${ }^{* * *} p<0.0001$, Wilcoxon's signed rank test.

trodes (Pearson's correlation; $r=0.1173 ; p=0.8022$ ) nor with the electrophysiological recording site in the $\mathrm{SNr}(r=0.4657 ; p=$ 0.2922).

\section{Overall results in kindled rats}

Effect of VPA on ADTs and seizure parameters in kindled rats Only animals with at least four VPA trials were considered for evaluation of the anticonvulsant efficacy of VPA on ADTs in kindled rats. For evaluation of ADTs and seizure parameters at ADT (seizure severity, seizure duration, afterdischarge duration), the individual mean of control values was compared with the individual mean of drug values for each individual animal.

Group mean \pm SEM of control ADTs was 72.0 $\pm 6.1 \mu \mathrm{A}$ (range, 29.2-145.0 $\mu \mathrm{A}$ ). VPA significantly increased ADTs in all animals tested (mean \pm SEM, $216.0 \pm 17.8 \mu \mathrm{A}$; range, 86.3$403.3 \mu \mathrm{A}$; paired $t$ test, $p<0.0001$ ). Seizure severity, seizure duration, and afterdischarge duration at ADT were significantly reduced after injection of VPA (paired $t$ test, $p<0.0001$; Table 1 ). At control ADTs, kindled rats always showed a secondarily generalized seizure [stage 5; Racine (1972)]. After injection of VPA, a generalized seizure could not always be elicited. In other words, in only 11 animals (40.7\%) a GST could be determined in all VPA trials. Therefore, the GST was not evaluated for quantification of VPA response.

\section{VPA plasma levels in kindled rats}

VPA plasma level was determined after each VPA trial. Only rats with a VPA plasma level of at least $250 \mu \mathrm{g} / \mathrm{ml}$ were used for analysis of anticonvulsant activity (see Materials and Methods). Mean plasma levels of VPA $30 \mathrm{~min}$ after intraperitoneal injection of $200 \mathrm{mg} / \mathrm{kg}$ were $376 \pm 5.7 \mu \mathrm{g} / \mathrm{ml}$ (mean \pm SEM; range, 254$547 \mu \mathrm{g} / \mathrm{ml})$.

Side effects induced by VPA in kindled rats

For later verification of putative differences in VPA-induced side effects between poor and good responders, severity of ataxia, hypolocomotion/sedation, and muscle relaxation, amount of wet dog shakes, body temperature, and motor coordination were determined in 80 trials (20 rats, four trials per rat) at two time points after injection of VPA (200 mg/kg, i.p.).

Without drug administration, score for ataxia, hypolocomotion/sedation, and muscle relaxation in kindled rats was 0 . Score of mean severity (range) of side effects 10 and 23 min after injection of VPA, respectively, was as follows: ataxia, $1.5(0-3)$ and 1.1 (0-3); hypolocomotion/sedation, $0.5(0-2)$ and $0.7(0-2)$; muscle relaxation, $1.7(0-3)$ and $1.6(0-2)$. Between injection of VPA and the electrical stimulation, animals showed $11.3(0-30)$ wet dog shakes. Body temperature was $38.2^{\circ} \mathrm{C}\left(36.8-39.1^{\circ} \mathrm{C}\right)$ before injection of $\mathrm{VPA}, 38.9^{\circ} \mathrm{C}\left(37.9-39.6^{\circ} \mathrm{C} ; p=0.0001\right.$, paired $t$ test $)$
10 min after injection of VPA, and $39.1^{\circ} \mathrm{C}\left(38.7-39.5^{\circ} \mathrm{C} ; p<\right.$ 0.0001 , paired $t$ test) $25 \mathrm{~min}$ after injection of VPA. In all of the four trials, at least 19 of 20 animals passed the rotarod task in one of three attempts at 10 and $23 \mathrm{~min}$ after VPA injection, respectively.

\section{Effect of VPA on spontaneous discharge rates}

Electrophysiological data from seven animals (one neuron per animal; Fig. $2 B$, rhombs; Fig. $3 C$, white bars) could be used for analysis of spontaneous discharge rate and pattern of SNr neurons recorded in kindled rats $1 \mathrm{~d}$ after a generalized kindled seizure. The time course of spikes (Fig. 4) represented the electrophysiological characteristics of nondopaminergic, presumably GABAergic nigral neurons as described in Materials and Methods [i.e., a biphasic (positive-negative) time course with an overall duration of $\sim 0.6-1.5 \mathrm{~ms}]$.

Intravenously injected VPA $(100 \mathrm{mg} / \mathrm{kg})$ caused a rapid reduction of firing rates in all recorded SNr neurons (Fig. 4A). Predrug levels of SNr discharge rates were 19.9 $\pm 2.1 \mathrm{~Hz}$ (mean \pm SEM; range, 8.8-25.1 Hz; Table 2). These predrug discharge rates were not correlated with the postkindling (control) ADTs (Table 2). VPA significantly reduced $\mathrm{SNr}$ discharge rates to $11.5 \pm 2.1$ $\mathrm{Hz}$ (mean \pm SEM; range, $4.4-20.8 \mathrm{~Hz} ; p=0.0046$; paired $t$ test) [i.e., maximum reduction of $\mathrm{SNr}$ discharge rates within $20 \mathrm{~min}$ after injection of VPA was $42.3 \pm 8.1 \%$ (mean \pm SEM; range, 17.0-79.4\%; Table 2)].

Control injections of saline during electrophysiological measurements in four animals (Fig. $2 \mathrm{~B}$, half-filled circle and open circles) did not significantly reduce $\mathrm{SNr}$ discharge rates (mean basal rate, $19.9 \pm 1.7 \mathrm{~Hz}$; range, $17.0-23.8 \mathrm{~Hz}$; mean rate after saline injection, $17.9 \pm 2.0 \mathrm{~Hz}$; range, $13.0-22.2 \mathrm{~Hz}$; paired $t$ test, $p=0.1072$ )

We did not determine VPA plasma levels during the electrophysiological experiments, because these levels were known from a previous study (Löscher et al., 1995). Although the intravenous dose of VPA $(100 \mathrm{mg} / \mathrm{kg})$ chosen for the electrophysiological experiments in anesthetized rats was lower than the intraperitoneal dose $(200 \mathrm{mg} / \mathrm{kg})$ used for determining the anticonvulsant effect, similar peak plasma concentrations of VPA (at least 250 $\mu \mathrm{g} / \mathrm{ml}$ ) are observed at time of testing. Thus, after intravenous injection of $100 \mathrm{mg} / \mathrm{kg}$, VPA reaches plasma concentrations of at least $250 \mu \mathrm{g} / \mathrm{ml}$ shortly ( $5 \mathrm{~min}$ ) after injection (Löscher et al., 1995; Rohlfs et al., 1996). In additional experiments, we found that, following peak levels, plasma levels stay on a plateau level for $\sim 20$ min before they drop down to $\sim 130 \mu \mathrm{g} / \mathrm{ml}$ (mean of five rats) $40 \mathrm{~min}$ after intravenous injection of $100 \mathrm{mg} / \mathrm{kg} \mathrm{VPA}$ (not illustrated). This is in line with the dose-dependent nonlinear 
A

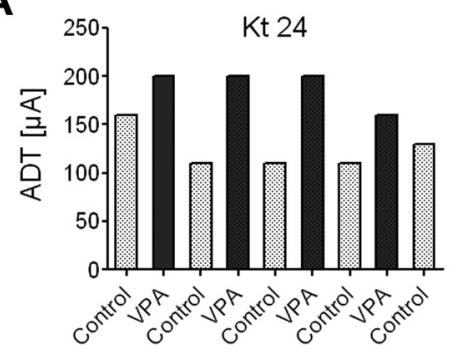

B

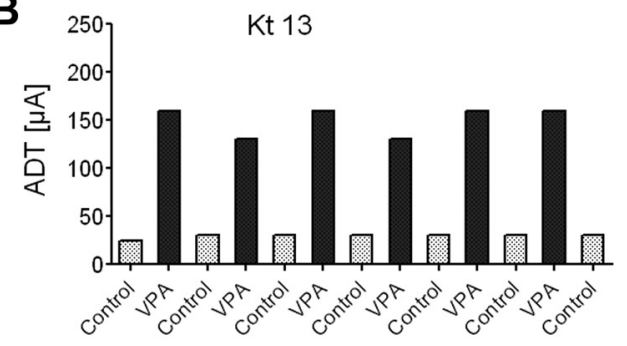

C



Figure 3. Mean individual increase of afterdischarge threshold after injection of valproate. Individual control thresholds as well as individual responses to VPA were reproducible in each rat from trial to trial and not just a chance event, indicating that the individual response to VPA was a characteristic feature of the animal. $\boldsymbol{A}$, Control thresholds and subsequent ADTs after intraperitoneal injection of $200 \mathrm{mg} / \mathrm{kg}$ VPA in a poor responder. $\boldsymbol{B}$, Control thresholds and subsequent ADTs after VPA injection in a good responder. The animal names are given in $\boldsymbol{A}$ and $\boldsymbol{B}$ for comparison with $\boldsymbol{C}$. C, Individual means of at least four drug values were compared with the individual mean of at least four predrug (control) values for each animal. When all animals were tested ( $n=27$ ), relative ADTs determined 30 min after VPA injection were significantly higher compared with predrug values (mean increase \pm SEM; $234 \pm 31.0 \%$ of predrug values; calculated by averaging all individual percentage ADT increases; paired $t$ test, $p<0.0001$ ). Mean group increase of ADTs (234\%) is indicated in the figure by the horizontal dotted line. Marked interindividual differences in the relative ADT increase in response to VPA were observed (range, 25.0 - 640.7\% above predrug values), revealing good (above average increase of $234 \%$ after VPA) and poor $(<234 \%)$ responders to VPA. After testing of VPA response in kindled rats, in vivo single-unit recordings were performed and evaluated in seven of these animals (white bars) within $14 \mathrm{~d}$ after the last VPA injection and $24 \mathrm{~h}$ after a kindled secondarily generalized seizure.

pharmacokinetics of VPA described previously for rats (Dickinson et al., 1979).

Effect of VPA on spontaneous discharge pattern (regularity) The regularity of SNr firing before and after systemic application of VPA was analyzed based on the ISIH parameters coefficient of variation, kurtosis, and skewness. Kurtosis and skewness were significantly changed toward a more regular neuronal discharge pattern after injection of VPA compared with predrug values (Table 3). Means \pm SEM for kurtosis were 21.2 \pm 2.9 before and $11.1 \pm 2.2$ after VPA injection; means \pm SEM for skewness were $3.8 \pm 0.3$ before and $2.7 \pm 0.3$ after VPA injection (Wilcoxon's signed rank test, $p=0.0156$ for both comparisons). The coefficient of variation was not significantly altered by VPA (Table 3 ).
Injections of saline during electrophysiological measurements did not significantly change ISIH parameters (Wilcoxon's signed rank test, $p=0.375$ for skewness and coefficient of variation, respectively; $p=0.625$ for kurtosis).

\section{Effect of VPA on spontaneous discharge pattern (burstiness} and periodicity)

Using the method described by Kaneoke and Vitek (1996) for evaluation of bursting and oscillatory properties, we detected bursts and oscillations in predrug discharge patterns in six of seven rats. The effect of VPA both on burstiness and on periodicity of $\mathrm{SNr}$ discharge patterns was inconsistent (summarized in Table 4).

Only neurons with bursts $(n=4)$ or oscillations $(n=5)$, respectively, were used for statistical analysis of the effect of VPA on spontaneous SNr burstiness and oscillation parameters. Significant differences between predrug and postdrug values were found neither for burstiness parameters (i.e., burst periods per second, burst periods per 1000 spikes, mean number of spikes in an burst, mean ISI of a burst period) nor for the dominant oscillation frequency (Table 4), assuming that the injection of VPA did not lead to a significant change in bursting and oscillatory properties of SNr neurons.

\section{Results in poor and good VPA responders}

Effects of VPA on ADTs and seizure parameters in kindled rats To compare the efficacy of VPA between different animals (i.e., ADT increase after VPA), individual means of at least four control ADTs were set at $100 \%$ and compared with the individual mean of at least four ADTs determined after injection of VPA for each individual animal. As described above, VPA significantly increased ADTs in all animals tested (range, 25-640\%). Marked interindividual differences in the extent of ADT increases in response to VPA revealing good responders (individual ADT increase above mean group increase of ADT of 234\%) and poor responders (below mean group increase of ADT) to VPA were observed (Fig. 3C). A poor (Fig. 3A) or good (Fig. 3B) response to VPA was reproducible in each rat and not just a chance event, indicating that the individual response to VPA was a characteristic feature of the animal. Seizure severity, seizure duration, and afterdischarge duration at ADT were significantly reduced after injection of VPA compared with respective seizure parameters of control trials (Wilcoxon's signed rank test; $p<0.0001$; Table 1 ), but there was no correlation between the magnitude of ADT increase in response to VPA and the extent of reduction of seizure parameters (not illustrated).

There were no significant correlations between the extent of ADT increase after VPA injection in individual rats and parameters accessed during kindling development [i.e., initial ADT, number of days until first stage 5 seizure, number of days until fully kindled state, cumulative seizure duration, and cumulative afterdischarge duration, respectively, for stages 2, 4, and 5 (not illustrated)]. In other words, a good or poor response to VPA was not related to prekindling seizure thresholds and kindling rates.

However, good responders tended to have lower control ADTs (compare Fig. $3 B$ ) than poor responders (Fig. $3 A$ ). We therefore analyzed group differences between rats arbitrarily allocated to good and poor responders as shown in Figure $3 C$. As shown in Table 5, the initial (prekindling) ADT did not differ between groups, while postkindling (control) ADT was significantly lower in good versus poor responders. Kindling rate (calculated as number of daily stimulations to first stage 5 seizure) or the cumulative afterdischarge duration until stage 5 did not differ 
A

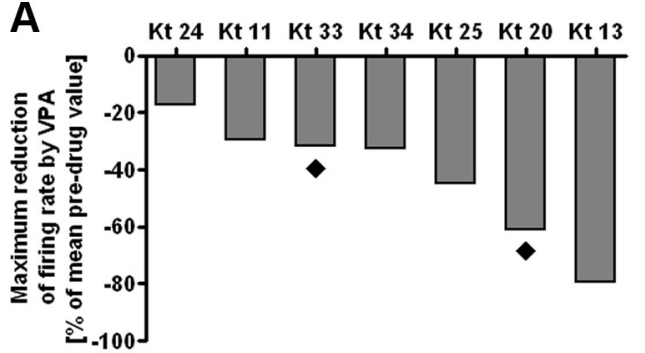

B

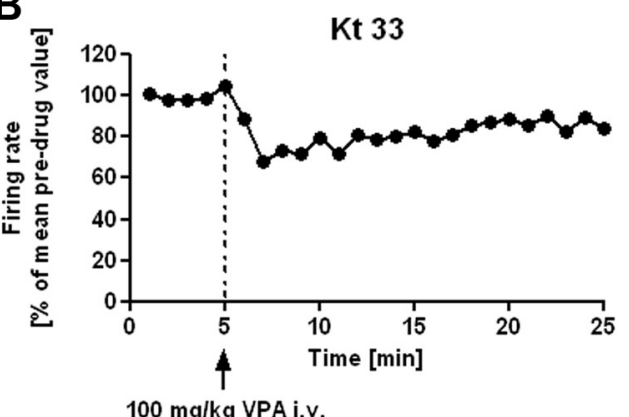

C

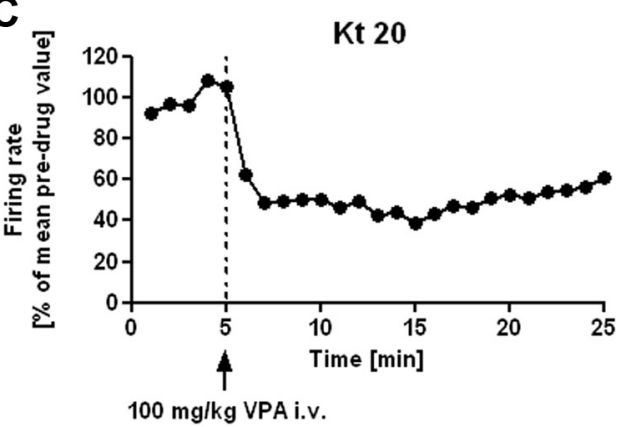

Table 2. Control afterdischarge thresholds and SNr baseline firing rates and correlation with SNr response to valproate

\begin{tabular}{llll}
\hline & $\begin{array}{l}\text { Control ADT (in } \\
\text { fully kindled } \\
\text { rats) }(\mu \mathrm{A})\end{array}$ & $\begin{array}{l}\text { SNr baseline } \\
\text { (predrug) firing } \\
\text { rate }(\mathrm{Hz})\end{array}$ & $\begin{array}{l}\text { SNr responsiveness** } \\
\text { (reduction of } \\
\text { firing rate by } \\
\text { valproate) }(\%)\end{array}$ \\
\hline Ktimal 24 & 122.5 & 25.1 & -17.0 \\
Kt 11 & 131.7 & 17.4 & -29.7 \\
Kt 33 & 82.5 & 22.8 & -31.7 \\
Kt 34 & 110.0 & 20.3 & -32.7 \\
Kt 25 & 42.0 & 8.8 & -44.8 \\
Kt 20 & 64.0 & 23.2 & -61.0 \\
Kt 13 & 29.2 & 21.4 & -79.4 \\
Median & 82.5 & 21.4 & -32.7 \\
Mean \pm SEM & $83.1 \pm 15.1$ & $19.9 \pm 2.1$ & $-42.3 \pm 8.1$
\end{tabular}

Seven animals could be used for evaluation of recordings of $\mathrm{SNr}$ neurons. There was no correlation between baseline (predrug) SNr firing rates and postkindling (control) ADTs determined after injection of saline (Pearson's correlation; $r=0.3095 ; p=0.4994)$. There was further no correlation between baseline SNr firing rates and the extent of reduction of SNr discharge rates by systemic application of VPA (Pearson's correlation; $r=0.5944 ; p=0.1593$ ). However, the reduction of SNr firing rate by VPA was significantly negatively correlated with the control ADTs (Pearson's correlation; $r=-0.8432 ;{ }^{* *} p=0.0172$ ). In other words, the lower the control ADT, the more marked VPA reduced SNr firing.

Table 3. Effect of valproate on the regularity of SNr firing

\begin{tabular}{lrrr}
\hline ISIH parameters & \multicolumn{1}{c}{ Predrug } & After VPA & \multicolumn{1}{c}{$p$} \\
\hline Kurtosis & $21.2 \pm 2.9$ & $11.1 \pm 2.2^{*}$ & 0.0156 \\
Skewness & $3.8 \pm 0.3$ & $2.7 \pm 0.3^{*}$ & 0.0156 \\
Coefficient of variation & $1.2 \pm 0.1$ & $1.1 \pm 0.8$ & 0.3750 \\
\hline
\end{tabular}

The regularity of firing of SNr neurons before and after systemic application of VPA was analyzed based on the ISIH parameters coefficient of variation, kurtosis, and skewness. Kurtosis and skewness were significantly changed (marked by asterisk) after injection of VPA compared to predrug values indicating a change towards a more regular neuronal discharge pattern caused by VPA. The coefficient of variation was not significantly altered by VPA (mean \pm SEM; Wilcoxon's signed rank test; $n=7$ ).

significantly between groups (Table 5 ). The average ADT following VPA (increase in percentage of individual control ADT) was significantly (more than three times) higher in good than in poor responders (Table 5).

VPA plasma levels in kindled rats

To verify that individual differences in response to VPA were not due to differences in VPA plasma levels, the amount of VPA in plasma was determined after each VPA trial. There was no correlation between ADT increase and plasma level of VPA in the trials evaluated ( $n=121$; Spearman's correlation, $r=0.0849$, $p=$ $0.3545)$, meaning that the individual response to VPA was not due to interindividually different plasma levels. This is also illustrated by the average VPA concentration determined in good and poor responders, which was about the same in both groups (Table 5).

Side effects induced by VPA in kindled rats

To verify whether a good or poor response to VPA is a general characteristic of the brain (i.e., is reflected on the severity of VPAinduced side effects), we evaluated putative correlations between side effects and anticonvulsant response to VPA. There was no correlation between the severity of ataxia, hypolocomotion/sedation, abdominal muscle tone decrease, or body temperature measured 10 and $23 \mathrm{~min}$ after VPA injection, respectively, and the individual anticonvulsant response to VPA in kindled rats $(n=$ 20 , mean of four trials per rat; Table 6). Wet dog shakes were counted continuously beginning immediately after the VPA injection and ending with current stimulation. There was no correlation between the mean individual number of wet dog shakes and the individual anticonvulsant response to VPA in kindled 
Table 4. Effect of valproate on burstiness and periodicity of SNr firing

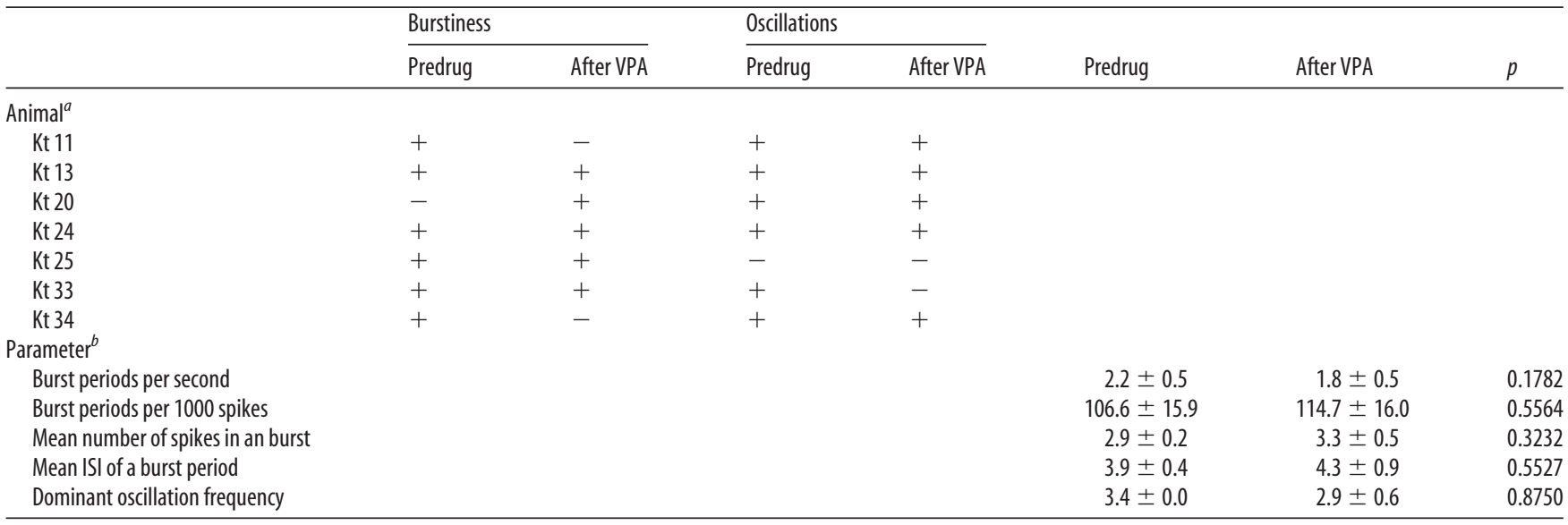

Bursts and oscillations were present in six of seven predrug discharge patterns.

${ }^{a}$ The effect of VPA on the burstiness as well as on the periodicity of discharge patterns of $\mathrm{SNr}$ neurons was inconsistent (+ , present; - , absent).

${ }^{b}$ Only neurons with bursts $(n=4)$ or oscillations $(n=5)$, respectively, were used for further statistical analysis. No significant differences were found for burstiness parameters or for the dominant oscillation frequency (mean \pm SEM; Wilcoxon's signed rank test before and after systemic application of VPA).

Table 5. Kindling parameters and effect of valproate in good and poor valproate responders

\begin{tabular}{|c|c|c|c|}
\hline Parameter & Poor responders & Good responders & $p$ \\
\hline \multicolumn{4}{|l|}{ Kindling development } \\
\hline Initial ADT $(\mu \mathrm{A})$ & $190.6 \pm 28.6(110-590)$ & $159.1 \pm 23.3(90-330)$ & 0.2384 \\
\hline Kindling rate (number of stimulations until stage $V$ ) & $7.4 \pm 0.5(5-12)$ & $7.7 \pm 0.9(5-12)$ & 0.8011 \\
\hline Cumulative afterdischarge duration until stage V (sec) & $151.9 \pm 12.2(52-250)$ & $210.1 \pm 28.3(110-385)$ & 0.1747 \\
\hline \multicolumn{4}{|l|}{ Effect of VPA on ADTs in kindled rats } \\
\hline Control ADT $(\mu \mathrm{A})$ in fully kindled rats & $83.3 \pm 8.3(39-145)$ & $55.8 \pm 6.7(29-110)^{*}$ & 0.0246 \\
\hline VPA ADT (\% of individual control ADT) & $123.3 \pm 16.2(25-233)$ & $394.8 \pm 34.5(245-640)^{* * *}$ & $<0.0001$ \\
\hline VPA plasma level $(\mu \mathrm{g} / \mathrm{ml})$ & $383.7 \pm 10.0(320-461)$ & $380.5 \pm 14.9(303-441)$ & 0.7860 \\
\hline
\end{tabular}

For comparison of kindling development and the effect of VPA on ADTs, the group of 27 kindled rats was divided into poor responders (individual ADT increase after $200 \mathrm{mg} / \mathrm{kg}$ VPA, i.p., below mean group increase of ADT of $234 \% ; n=16$ ) and good responders (individual ADT increase $>234 \% ; n=11$ ). Good and poor responders did not differ in kindling development (Mann-Whitney $U$ test). Good and poor responders significantly differed in the predrug (control) ADT determined after injection of saline and in the effect of VPA on ADTs (Student's $t$ test; marked by asterisks). The average VPA concentration was about the same in good and poor responders (Mann-Whitney $U$ test). Data are means \pm SEM (range).

Table 6. Lack of correlation between the severity of side effects induced by valproate and the response to valproate in kindled rats

\begin{tabular}{|c|c|c|c|c|c|c|c|c|}
\hline \multirow[b]{2}{*}{ Time after injection of VPA } & \multicolumn{2}{|l|}{ Ataxia } & \multicolumn{2}{|c|}{ Hypolocomotion/sedation } & \multicolumn{2}{|c|}{ Muscle relaxation } & \multicolumn{2}{|c|}{ Increase in body temperature } \\
\hline & $r$ & $p$ & $r$ & $p$ & $r$ & $p$ & $r$ & $p$ \\
\hline $10 \mathrm{~min}$ & -0.1170 & 0.6234 & -0.06195 & 0.7953 & 0.2243 & 0.3418 & -0.3991 & 0.0813 \\
\hline $23 \mathrm{~min}$ & 0.2147 & 0.3634 & -0.1294 & 0.5865 & 0.1836 & 0.4385 & -0.04523 & 0.8498 \\
\hline
\end{tabular}

For evaluation of side effects, individual means of tests starting 10 and 23 min after VPA injection ( $200 \mathrm{mg} / \mathrm{kg}$, i.p.) were compared with the individual mean effect of VPA on ADTs in kindled rats ( $n=20)$. There was no correlation between the mean individual severity of ataxia, hypolocomotion/sedation, abdominal muscle tone decrease, or increase in body temperature after VPA injection and the mean individual response to VPA (Spearman's correlation).

rats. Rotarod performance also revealed no differences between good and poor VPA responders (both not illustrated).

Effect of VPA on spontaneous discharge rates

The VPA-induced reduction of SNr firing rates was individually more or less pronounced (Fig. $4 B$, poor responder; $C$, good responder), ranging from 17.0 to $79.4 \%$ maximum reduction of basal firing rates between the individual animals within $20 \mathrm{~min}$ after injection of VPA (Fig. 4A). This reduction in firing rate occurred rapidly in all investigated rats, independent of a good or poor response to VPA, and occasionally showed slow and moderate recovery (Fig. 4). According to our hypothesis, the extent of reduction of $\mathrm{SNr}$ discharge rates by systemically applied VPA (100 mg/kg, i.v.) exhibited a significant positive correlation with the extent of the anticonvulsant efficacy of VPA in kindled rats (Fig. 5A; Pearson's correlation; $r=0.8654 ; p=0.0119$ ).

Prekindling seizure thresholds and kindling rates did not influence later efficacy of VPA on SNr firing rate. There were no significant correlations between the extent of reduction of $\mathrm{SNr}$ firing rates by systemic VPA and parameters accessed during kin- dling development [i.e., initial ADT, days until first stage 5 seizure and until fully kindled state, cumulative seizure duration, and cumulative afterdischarge duration, respectively, for stages 3 , 4 , and 5 (not illustrated)]. The extent of reduction of $\mathrm{SNr}$ discharge rates by systemic VPA was further not correlated with seizure parameters recorded at ADT determinations [i.e., seizure stage, seizure duration, and afterdischarge duration (not illustrated)]. Also, the extent of reduction of SNr discharge rates by systemic VPA was not correlated with the baseline (predrug) SNr firing rates (Table 2). However, the extent of reduction of $\mathrm{SNr}$ firing rates by systemic VPA was significantly negatively correlated with the postkindling (control) ADTs [i.e., the lower the postkindling (control) ADT, the higher the ability of VPA to reduce SNr firing] (Table 2; Pearson's correlation; $r=-0.8432$; $p=0.0172$ ).

Effect of VPA on spontaneous discharge pattern (regularity) We observed a significant correlation between ADT increase and the change of skewness in the neuronal discharge pattern by VPA (Spearman's correlation; $r=-0.8214 ; p=0.0341$; Fig. $5 B$ ). In 
A
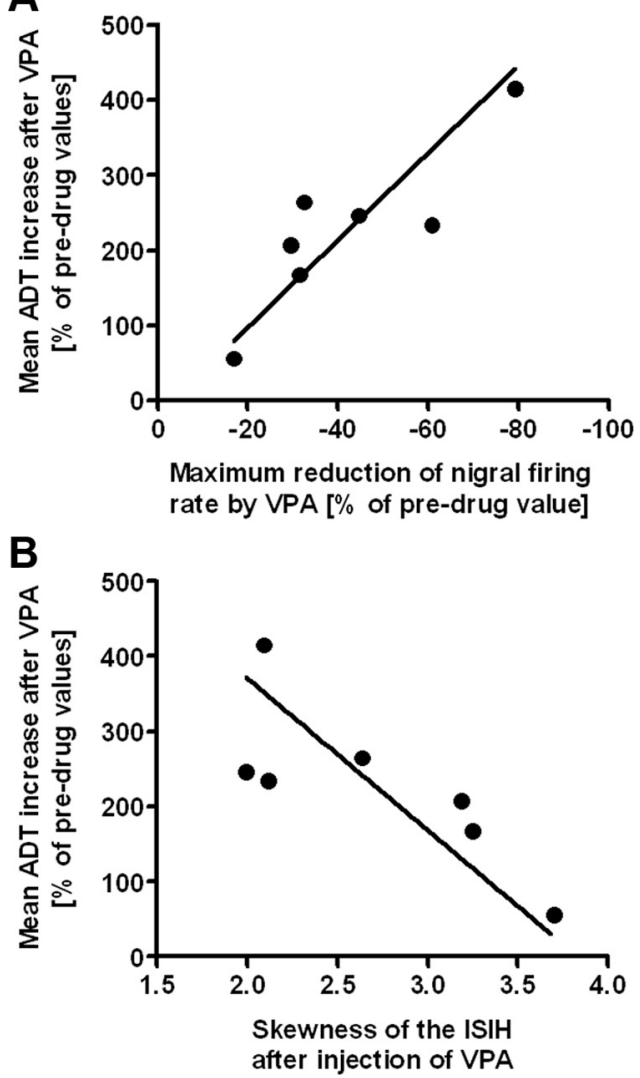

Figure 5. Correlation between the effect of valproate on afterdischarge thresholds in kindled rats and efficacy of valproate on $\mathrm{SNr}$ firing rate and pattern. $A$, The extent of reduction of discharge rates of $\mathrm{SNr}$ neurons by systemic application of VPA was significantly positively correlated with the extent of the anticonvulsant efficacy of VPA in kindled rats $(n=7)$ (i.e., ADT increase) (Pearson's correlation; $r=0.8654 ; p=0.0119$ ). In other words, the more marked VPA raised ADTs, the more marked was the reduction in SNr firing rate by VPA. $\boldsymbol{B}$, High skewness values reflect irregular firing while a skewness of zero reflects regular firing. The skewness of the ISIH of SNr firing after systemic application of VPA was significantly negatively correlated with the extent of the anticonvulsant efficacy of VPA in kindled rats (i.e., ADT increase) $(n=7$; Spearman's correlation; $r=-0.8214 ; p=0.0341$ ). In other words, the more marked VPA raised ADTs, the more marked was VPA able to induce a regular firing pattern (mirrored as lowered skewness).

other words, the more pronounced VPA increased the ADT, the more pronounced was the VPA-induced regularity of SNr firing. However, there was no significant correlation between ADT increase and the change of kurtosis by VPA (not illustrated).

\section{Discussion}

The main findings of the present study were (1) that good and poor VPA responders can be selected in kindled rats and (2) that this good or poor response is neither correlated to VPA plasma levels nor to VPA-induced side effects, but (3) the anticonvulsant response is significantly correlated with the ability of VPA to reduce firing rates of nondopaminergic, presumably GABAergic $\mathrm{SNr}$ neurons and to induce a regular firing pattern of $\mathrm{SNr}$ neurons.

\section{Good and poor anticonvulsant response to VPA}

In the phenytoin nonresponder model described by Löscher and Rundfeldt (1991), kindled female Wistar rats were selected as responders or nonresponders based on their sensitivity to the anticonvulsant effect of a repeatedly administered high dose of phenytoin (75 mg/kg, i.p.). Löscher et al. (1993) showed that, in
$80 \%$ of phenytoin nonresponders, VPA ( $200 \mathrm{mg} / \mathrm{kg}$, i.p. $)$ was also not efficient to increase ADTs. We now successfully selected kindled rats as good and poor responders to VPA. As demonstrated by our data, this strategy is useful both for better understanding mechanisms of anticonvulsant action of AEDs and potential mechanisms of AED resistance.

We assume that poor VPA responders can be considered difficult-to-treat or pharmacoresistant, because their relatively weak ADT increase was obtained at a dose $(200 \mathrm{mg} / \mathrm{kg})$ that is associated with severe adverse effects. In other words, it is very likely that lower, better tolerable doses of VPA would have still increased ADT in good but not poor responders. Despite comparable plasma concentrations, rats responded differently to VPA, thus excluding differences in elimination rate of VPA as a cause of the interindividual differences in anticonvulsant response. Furthermore, good and poor VPA responders showed equal severity of VPA-induced side effects, indicating that mechanisms contributing to a good versus poor anticonvulsant response to VPA are different from those involved in side effects, and that a good or poor response is not a general characteristic of the brain.

Similar to phenytoin responders and nonresponders (Löscher et al., 1993), kindling development was not correlated to the later response to VPA in fully kindled rats. The only significant difference in kindling parameters was that the average postkindling control ADT was lower in good than poor VPA responders. Such a difference had previously also been observed in phenytoin responders versus nonresponders (Löscher et al., 1993) and, together with data from selective breeding of responders and nonresponders, had added to the hypothesis that the genetic background of an individual rat determines whether it becomes a responder or nonresponder to AEDs following the kindling process (Ebert and Löscher, 1999).

\section{Good and poor response of SNr neurons to VPA}

VPA has been shown to increase GABA synthesis in the $\mathrm{SNr}$, leading to inhibition of GABAergic projection neurons in this region (Löscher, 2002). Inhibition of GABAergic nigral neurons is thought to cause a disinhibition of nigral efferences in the midbrain and brainstem, which then can mediate anticonvulsant effects (Depaulis et al., 1994; Nolte et al., 2006; Gale et al., 2008). It was suggested before that suppression of spontaneous $\mathrm{SNr}$ neuronal firing may be one important mechanism through which VPA exerts its anticonvulsant properties (Kerwin et al., 1980; Waszczak et al., 1986; Farrant and Webster, 1989; Löscher et al., 1995; Rohlfs et al., 1996). The present findings using good and poor VPA responders substantiate this concept.

We previously observed that the efficacy of VPA to reduce $\mathrm{SNr}$ discharge rates was significantly decreased in amygdala-kindled rats compared with naive controls and concluded a kindlinginduced change of SNr sensitivity to VPA (Gernert et al., 2004). Amygdala kindling does not induce neurodegeneration in the SNr (Freichel et al., 2004), but amygdala-kindled rats differ from nonkindled controls in a reduced activity of the GABAsynthesizing enzyme GAD (glutamate decarboxylase), reduced nerve terminal (synaptosomal) GABA concentrations, and reduced GABA receptor binding in the SNr (Löscher and Schwark, 1985, 1987). This likely explains that kindled rats are less sensitive than nonkindled rats to the effects of VPA on the $\mathrm{SNr}$ (Gernert et al., 2004). Accordingly, interindividual differences in the effects of kindling on GABA neurochemistry in the $\mathrm{SNr}$ could be involved in the present findings. 
Interestingly, numerous studies showed that the properties of the $\mathrm{SNr}$ to modulate seizure activity are dependent on many different factors including rat strain (Moshé et al., 1994, 1995; Velísková et al., 1998, 2001; Gernert and Löscher, 2001; Galanopoulou et al., 2003; Velísek et al., 2005). Differences in genetic background are then likely also involved in different pharmacosensitivity of SNr neurons to VPA.

It is noteworthy that the $\mathrm{SNr}$ responsiveness to VPA was significantly negatively correlated with the postkindling control ADTs. In other words, the lower the control ADT, the higher was the SNr responsiveness to VPA. Together with the lower control ADTs in good responders, it seems that a higher seizure susceptibility of kindled rats entails a better efficacy of VPA (on kindled $\mathrm{ADT}$ as well as on $\mathrm{SNr}$ firing rate).

In addition to the effect of VPA on discharge rates, we observed a correlation between the anticonvulsant effect of VPA and its effect on SNr discharge pattern. The more the individual ADT in kindled rats was increased by VPA, the more marked was the ability of VPA to induce a regular SNr firing pattern in the respective rat. Our findings emphasize the hypothesis that, in the kindling model, an irregular neuronal discharge pattern reflects a pathological condition while a regular pattern resembles a physiological or therapeutically treated condition (Gernert et al., 2004; Kücker et al., 2010).

In the rat kindling model of TLE, kindling-induced network plasticity distant to the epileptic focus (i.e., in the basal ganglia) has been shown (Gernert et al., 2004; Nolte et al., 2006; Kücker et al., 2010). The network hypothesis suggests that "epileptic networks" in pharmacoresistant patients differ from those in pharmacosensitive patients. In our study, differences in kindling development in individual rats did not correlate with the subsequent effect of VPA on ADTs, suggesting that the same kindling process resulted in individually different kindling-induced network or target changes on the level of the SNr. Further studies hopefully will elucidate the main factors contributing to individual differences in VPA-induced suppression of SNr firing.

\section{Alternative explanations for a good or poor response of $\mathrm{SNr}$ neurons to VPA}

Because we could only record one $\mathrm{SNr}$ neuron per animal to investigate its response to systemically applied VPA, we cannot be sure that all SNr neurons of a specific animal respond in the same manner to VPA. In other words, it is conceivable that some neurons in the $\mathrm{SNr}$ do not respond to VPA, independent of its effect on ADT.

We have previously shown that the estrous cycle does not affect seizure susceptibility (Wahnschaffe and Löscher, 1992) or drug effects (Rundfeldt et al., 1990) in the kindling model of epilepsy. It is therefore rather unlikely that an individually different hormonal stage of the rats was responsible for differences in the response of SNr neurons to VPA.

A comparable vigilance state of the animals during recordings is important for assessment of individual differences in the response of neurons to VPA. Stability of heart rate, blood pressure, and expired $\mathrm{CO}_{2}$ level and the lack of episodic tachycardia indicated similar vigilance during our electrophysiological recordings. It is interesting to note that we previously compared different anesthetic protocols, including the one used in the present study, on their influence on the response of $\mathrm{SNr}$ neurons to VPA (Löscher et al., 1995). In this previous study, we could show that the VPA-induced percentage decrease of individual baseline firing rates did not differ between conscious rats and rats in which the fentanyl/gallamine protocol of the present study was used, while other protocols, such as the use of chloral hydrate, markedly influenced the response of SNr neurons to VPA. We therefore assume that depth of anesthesia is unlikely to explain differences in the response of SNr neurons to VPA.

Recording sites within the $\mathrm{SNr}$ were not correlated with the efficacy of VPA to reduce neuronal activity. Nevertheless, because of the low sample size, we cannot completely exclude that the recording sites within the $\mathrm{SNr}$ might have influenced the responsiveness to VPA. This is especially noteworthy because previous studies showed that kindling causes an increased firing rate only in the posterior part of the SNr and a reduced efficacy of VPA to lower firing rate only in the anterior $\mathrm{SNr}$ of adult female rats (Gernert et al., 2004). In other studies, therapeutic manipulation of the anterior rather than the posterior $\mathrm{SNr}$ was effective against flurothyl-induced clonic seizures in adult male rats (Moshé et al., 1995; Velísková et al., 1998).

\section{Concluding remarks}

Our study indicates that the effect of VPA on SNr activity is critically involved in its anticonvulsant efficacy and represents the first evidence that pharmacoresistance in a rat model for TLE is reflected on the level of basal ganglia network activity. The present evidence of a reflection of anticonvulsant AED responses on the level of the $\mathrm{SNr}$ adds to a better understanding of the pathophysiology of pharmacoresistant TLE and emphasizes the role of the basal ganglia as a target structure for new treatment options.

\section{References}

Albala BJ, Moshé SL, Okada R (1984) Kainic-acid-induced seizures: a developmental study. Brain Res 315:139-148.

Deniau JM, Hammond C, Riszk A, Feger J (1978) Electrophysiological properties of identified output neurons of the rat substantia nigra (pars compacta and pars reticulata): evidences for the existence of branched neurons. Exp Brain Res 32:409-422.

Depaulis A, Vergnes M, Marescaux C (1994) Endogenous control of epilepsy: the nigral inhibitory system. Prog Neurobiol 42:33-52.

De Sarro G, Meldrum BS, Reavill C (1984) Anticonvulsant action of 2-amino-7-phosphonoheptanoic acid in the substantia nigra. Eur J Pharmacol 106:175-179.

De Sarro G, Patel S, Meldrum BS (1986) Anticonvulsant action of a kainate antagonist gamma-D-glutamyl aminomethylsulphonic acid injected focally into the substantia nigra and entopeduncular nucleus. Eur J Pharmacol 132:229-236.

De Sarro G, De Sarro A, Meldrum BS (1991) Anticonvulsant action of 2-chloroadenosine injected focally into the inferior colliculus and substantia nigra. Eur J Pharmacol 194:145-152.

Dickinson RG, Harland RC, Ilias AM, Rodgers RM, Kaufman SN, Lynn RK, Gerber N (1979) Disposition of valproic acid in the rat: dose-dependent metabolism, distribution, enterohepatic recirculation and choleretic effect. J Pharmacol Exp Ther 211:583-595.

Ebert U, Löscher W (1999) Characterization of phenytoin-resistant kindled rats, a new model of drug-resistant partial epilepsy: influence of genetic factors. Epilepsy Res 33:217-226.

Ebert U, Rundfeldt C, Lehmann H, Löscher W (1999) Characterization of phenytoin-resistant kindled rats, a new model of drug-resistant partial epilepsy: influence of experimental and environmental factors. Epilepsy Res 33:199-215.

Farrant M, Webster RA (1989) Neuronal activity, amino acid concentration and amino acid release in the substantia nigra of the rat after sodium valproate. Brain Res 504:49-56.

Freichel C, Ebert U, Potschka H, Löscher W (2004) Amygdala-kindling does not induce a persistent loss of GABA neurons in the substantia nigra pars reticulata of rats. Brain Res 1025:203-209.

French JA (2007) Refractory epilepsy: clinical overview. Epilepsia 48 [Suppl 1]:3-7.

Galanopoulou AS, Kyrozis A, Claudio OI, Stanton PK, Moshé SL (2003) Sex-specific KCC2 expression and $\mathrm{GABA}_{\mathrm{A}}$ receptor function in rat substantia nigra. Exp Neurol 183:628-637. 
Gale K, Proctor M, Velísková J, Nehlig A (2008) Basal ganglia and brainstem anatomy and physiology. In: Epilepsy: a comprehensive textbook (Engel JJ, Pedley TA, eds), pp 367-384. Philadelphia: Lippincott Williams and Wilkins.

Garcia-Cairasco N, Sabbatini RM (1983) Role of the substantia nigra in audiogenic seizures: a neuroethological analysis in the rat. Braz J Med Biol Res 16:171-183.

Gernert M, Löscher W (2001) Lack of robust anticonvulsant effects of muscimol microinfusions in the anterior substantia nigra of kindled rats. Eur J Pharmacol 432:35-41.

Gernert M, Fedrowitz M, Wlaz P, Löscher W (2004) Subregional changes in discharge rate, pattern, and drug sensitivity of putative GABAergic nigral neurons in the kindling model of epilepsy. Eur J Neurosci 20:2377-2386.

Grace AA, Bunney BS (1979) Paradoxical GABA excitation of nigral dopaminergic cells: indirect mediation through reticulata inhibitory neurons. Eur J Pharmacol 59:211-218.

Guyenet PG, Aghajanian GK (1978) Antidromic identification of dopaminergic and other output neurons of the rat substantia nigra. Brain Res 150:69-84.

Iadarola MJ, Gale K (1982) Substantia nigra: site of anticonvulsant activity mediated by gamma-aminobutyric acid. Science 218:1237-1240.

Kaneoke Y, Vitek JL (1996) Burst and oscillation as disparate neuronal properties. J Neurosci Methods 68:211-223.

Kerwin RW, Olpe HR, Schmutz M (1980) The effect of sodium- $n$-dipropyl acetate on gamma-aminobutyric acid-dependent inhibition in the rat cortex and substantia nigra in relation to its anticonvulsant activity. $\mathrm{Br} \mathrm{J}$ Pharmacol 71:545-551.

Kücker S, Töllner K, Piechotta M, Gernert M (2010) Kindling as a model of temporal lobe epilepsy induces bilateral changes in spontaneous striatal activity. Neurobiol Dis 37:661-672.

Le Gal La Salle G, Kaijima M, Feldblum S (1983) Abortive amygdaloid kindled seizures following microinjection of gamma-vinyl-GABA in the vicinity of substantia nigra in rats. Neurosci Lett 36:69-74.

Löscher W (1989) Valproate enhances GABA turnover in the substantia nigra. Brain Res 501:198-203.

Löscher W (2002) Basic pharmacology of valproate: a review after 35 years of clinical use for the treatment of epilepsy. CNS Drugs 16:669-694.

Löscher W (2006) Animal models of drug-refractory epilepsy. In: Models of seizures and epilepsy (Pitkänen A, Schwartzkroin PA, Moshe SL, eds), pp 551-567. Burlington, MA: Academic.

Löscher W (2007) The pharmacokinetics of antiepileptic drugs in rats: consequences for maintaining effective drug levels during prolonged drug administration in rat models of epilepsy. Epilepsia 48:1245-1258.

Löscher W, Potschka H (2005) Drug resistance in brain diseases and the role of drug efflux transporters. Nat Rev Neurosci 6:591-602.

Löscher W, Rundfeldt C (1991) Kindling as a model of drug-resistant partial epilepsy: selection of phenytoin-resistant and nonresistant rats. J Pharmacol Exp Ther 258:483-489.

Löscher W, Schwark WS (1985) Evidence for impaired GABAergic activity in the substantia nigra of amygdaloid kindled rats. Brain Res 339: $146-150$.

Löscher W, Schwark WS (1987) Further evidence for abnormal GABAergic circuits in amygdala-kindled rats. Brain Res 420:385-390.

Löscher W, Rundfeldt C, Hönack D (1993) Pharmacological characterization of phenytoin-resistant amygdala-kindled rats, a new model of drugresistant partial epilepsy. Epilepsy Res 15:207-219.

Löscher W, Rohlfs A, Rundfeldt C (1995) Reduction in firing rate of substantia nigra pars reticulata neurons by valproate: influence of different types of anesthesia in rats. Brain Res 702:133-144.
McNamara JO, Galloway MT, Rigsbee LC, Shin C (1984) Evidence implicating substantia nigra in regulation of kindled seizure threshold. J Neurosci 4:2410-2417.

Moshé SL, Albala BJ (1984) Nigral muscimol infusions facilitate the development of seizures in immature rats. Brain Res 315:305-308.

Moshé SL, Sperber EF, Brown LL, Tempel A (1992) Age-dependent changes in substantia nigra GABA-mediated seizure suppression. Epilepsy Res Suppl 8:97-106.

Moshé SL, Brown LL, Kubová H, Velísková J, Zukin RS, Sperber EF (1994) Maturation and segregation of brain networks that modify seizures. Brain Res 665:141-146.

Moshé SL, Garant DS, Sperber EF, Velísková J, Kubova H, Brown LL (1995) Ontogeny and topography of seizure regulation by the substantia nigra. Brain Dev 17 [Suppl]:61-72.

Nolte MW, Löscher W, Gernert M (2006) Pedunculopontine neurons are involved in network changes in the kindling model of temporal lobe epilepsy. Neurobiol Dis 23:206-218.

Paxinos G, Watson C (2007) The rat brain in stereotactic coordinates, Ed 6. Amsterdam: Academic.

Potschka H, Löscher W (2001) In vivo evidence for P-glycoproteinmediated transport of phenytoin at the blood-brain barrier of rats. Epilepsia 42:1231-1240.

Racine RJ (1972) Modification of seizure activity by electrical stimulation. II. Motor seizure. Electroencephalogr Clin Neurophysiol 32:281-294.

Remy S, Beck H (2006) Molecular and cellular mechanisms of pharmacoresistance in epilepsy. Brain 129:18-35.

Rohlfs A, Rundfeldt C, Koch R, Löscher W (1996) A comparison of the effects of valproate and its major active metabolite E-2-en-valproate on single unit activity of substantia nigra pars reticulata neurons in rats. J Pharmacol Exp Ther 277:1305-1314.

Rundfeldt C, Hönack D, Löscher W (1990) Phenytoin potently increases the threshold for focal seizures in amygdala-kindled rats. Neuropharmacology 29:845-851.

Schmidt D, Löscher W (2005) Drug resistance in epilepsy: putative neurobiologic and clinical mechanisms. Epilepsia 46:858-877.

Simons DJ, Land PW (1987) A reliable technique for marking the location of extracellular recording sites using glass micropipettes. Neurosci Lett $81: 100-104$.

Sperber EF, Wong BY, Wurpel JN, Moshé SL (1987) Nigral infusions of muscimol or bicuculline facilitate seizures in developing rats. Brain Res 465:243-250.

Szoeke CE, Newton M, Wood JM, Goldstein D, Berkovic SF, O’Brien TJ, Sheffield LJ (2006) Update on pharmacogenetics in epilepsy: a brief review. Lancet Neurol 5:189-196.

Velísek L, Velísková J, Ravizza T, Giorgi FS, Moshé SL (2005) Circling behavior and $\left[{ }^{14} \mathrm{C}\right] 2$-deoxyglucose mapping in rats: possible implications for autistic repetitive behaviors. Neurobiol Dis 18:346-355.

Velísková J, Löscher W, Moshé SL (1998) Regional and age specific effects of zolpidem microinfusions in the substantia nigra on seizures. Epilepsy Res 30:107-114

Velísková J, Liptáková S, Hussain S (2001) The effects of $N$-methyl-D-aspartate antagonist 2-amino-7-phosphonoheptanoic acid microinfusions into the adult male rat substantia nigra pars reticulata are site-specific. Neurosci Lett 316:108-110.

Wahnschaffe U, Löscher W (1992) Lack of changes in seizure susceptibility during the estrous cycle in kindled rats. Epilepsy Res 13:199-204.

Waszczak BL, Lee EK, Walters JR (1986) Effects of anticonvulsant drugs on substantia nigra pars reticulata neurons. J Pharmacol Exp Ther 239:606611. 